\title{
Unusual Clinical and Biochemical Presentations of Severe Covid-19 Pneumonia Managed in General Ward
}

\author{
QAA RAHMAN $^{\mathrm{a}}$, HAMN AHASAN ${ }^{\mathrm{b}}, \mathrm{T}_{\mathrm{CHOWDHURY}}^{\mathrm{c}}$, IB REZA ${ }^{\mathrm{d}}, \mathrm{QT}^{\mathrm{Q}}$ ISLAM $^{\mathrm{e}}$
}

\begin{abstract}
:
Covid-19 is a multi-system infection which predominately infects the lungs. This new disease can present as an asymptomatic infection to life threatening infections leading to death, making it a challenge for healthcare workers across the globe in this pandemic. Covid-19 pneumonia often shows a set pattern of symptom progression along its disease course, although the timing of each symptom may vary in different individuals. In this case report, we will discuss the unusual presentation of a middle-aged man who suffered a prolonged course of disease and who was managed on suspicion of a secondary bacterial infection along with cytokine storm. The logical, thoughtful
\end{abstract}

Introduction:

Coronavirus disease 2019 (Covid-19) is an infectious disease caused by severe acute respiratory syndrome coronavirus 2 (SARS-CoV-2).It was first identified in December 2019 in Wuhan, Hubei, China, and has resulted in an ongoing pandemic.

Because it is a new disease, it is important that we know about this disease in detail. SARS-CoV-2 invades host human cells by binding to the ACE2 receptor ${ }^{1}$. It spreads mainly through saliva, droplets, or discharges from the nose of an infected individual after coughing or sneezing. The incubation period

a. Quazi Audry Arafat Rahmana, Asstt. Registrar of Medicine, Popular Medical College

b. HAM Nazmul Ahasan, Professor of Medicine, Popular Medical College

c. Tasmina Chowdhury, Registrarof Medicine, Popular Medical College

d. Ishrat Binte Reza, Asstt. Professor of Medicine, Popular Medical College

e. Quazi Tarikul Islam, Professor of Medicine, Popular Medical College

Address of Correspondence: Dr. Quazi Audry Arafat Rahman, MBBS, Asst. Registrar of Medicine, Popular Medical College, Email: audryrahman@gmail.com

Received: 01 September, 2020

Accepted: 13 September, 2020 management options that we chose at various stages of his disease in general ward without use of immune modulatory drug like tocilizumab,which eventually led to his cure are also discussed here.Persistence of positive RT-PCR in the $4^{\text {th }}$ week is also seen in this case.Emphasis is given on how these tricky cases can be dealt in the general ward without shifting them in the $\mathrm{HDU}$, where $\mathrm{HDU}$ or ICU facilities are not available or affordable.

Keywords: Covid-19, bacterial infection, cytokine storm, lactate, antibiotics, dexamethasone, remdisivir, tocilizumab, RT-PCR.

(J Bangladesh Coll Phys Surg 2020; 38: 223-228)

DOI: https://doi.org/10.3329/jbcps.v38i4.48986

can range from 2-14 days ${ }^{2}$. Although it is well documented that Covid-19 is primarily manifested as a respiratory tract infection, emerging data indicate that it should be regarded as a systemic disease involving multiple systems, including cardiovascular, respiratory, gastrointestinal, neurological, hematopoietic and immune system ${ }^{3}$. Symptoms of Covid-19 are variable, but usually include fever and a cough.

Covid-19 infection is accompanied by an aggressive inflammatory response with the release of a large amount of pro-inflammatory cytokines in an event known as cytokine storm. The cytokine storm results from a sudden acute increase in circulating levels of different pro-inflammatory cytokines including IL-6, IL-1, TNF- $\alpha$, and interferon.This increase in cytokines results in influx of various immune cells from the circulation into the site of infection with destructive effects on human tissue resulting from destabilization of endothelial cell to cell interactions, damage of vascular barrier, capillary damage, diffuse alveolar damage, multiorgan failure, and ultimately death. Lung injury is one consequence of the cytokine storm that can progress into acute lung injury or its more severe form $\mathrm{ARDS}^{4}$. Markers such 
as IL-6, serum ferritin, CRP and D-dimer can be done to look for evidence of cytokine storm.

The course of this disease may range from a few days up to a few weeks. While some cases may recover within 10 - 14 days, other cases may have a prolonged course. RT-PCR may also remain positive for Covid-19 for more than 4 weeks. However, the clinical importance of positive RT-PCR after $3^{\text {rd }}$ week is questionable as such instances usually imply the presence of dead RNA strand in the body instead of having active replicating viruses.

\section{Case Report:}

A 48-year-old, normotensive, non-diabetic male, brought himself to Popular Medical College Hospital with cough, fever and late onset dyspnoea. Initially, he had dry cough 16 days prior to admission which was not associated with any dyspnoea. He used to monitor his $\mathrm{SpO}_{2}$ regularly, which was always more than $95 \%$ at room air. He developed low grade fever 4 days later. Fever was continued in nature which subsided upon taking paracetamol. His highest recorded temperature before admission was $101^{\circ} \mathrm{F}$. At the onset of fever, he also had loose motion for 3 days. The patient was given Tab.Moxaclav (Amoxicillin+Clavulanic acid combination) for 5 days and a single dose of Tab Ivermectin $12 \mathrm{mg}$ by local doctors from Day 6 of symptom onset. Having high suspicion of Covid-19 during this pandemic, he was given a CXR and RT-PCR for Covid-19 on 27/7/2020 (Day 10 from symptom onset)by local physicians. RT-PCR report came positive. However, CXR showed normal findings. Next day, new-onset mild dyspnea started along with the fever and cough, so he continued to take the antibiotics on his own. Dyspnea was initially within acceptable limit, but soon he found himself distressed and struggled to complete sentences in one breath. So, he decided to get admitted.

Patient was admitted in Covid unit on 2/8/2020 (Day 16 of his illness). On admission, his temperature was $101^{\circ} \mathrm{F}$, pulse rate $130 \mathrm{~b} / \mathrm{min}, \mathrm{BP} 125 / 75 \mathrm{mmHg}$ and respiratory rate $22 / \mathrm{min}, \mathrm{SpO}_{2}$ was $94 \%$ at room air and $2 \mathrm{~L}$ of Oxygen was given. Baseline investigations were given which showed leukocytosis $\left(17.4 \mathrm{~K} / \mathrm{mm}^{3}\right)$ and raised CRP $(86 \mathrm{mg} / \mathrm{L})$ along with bilateral lung involvement on CXR. Considering the likelihood of developing secondary bacterial infection, Inj. Meropenem was started. Therapeutic dose of LMWH (60 mg 12 hourly) and Inj. Dexamethasone $10 \mathrm{mg} 12$ hourly were also started on admission (Day 16). Another CXR was done the next day (Day 17)which showed increasing bilateral lung involvement (Fig:1).His oxygen requirement also increased. So, Remdisivir was started that day.CBC and other investigations including infective markers were done on subsequent days which showed very high WBC count, lactate and LDH which suggested an underlying secondary bacterial infection with sepsis along with cytokine storm with the high ferritin (Table: I). However, growth of bacteria by culture could not be confirmed as no organisms were found on culture, which was attributed to his early use of antibiotics at home.SGPT was raised at $124 \mathrm{U} / \mathrm{L}$ (Day 19). However, his D-dimer was always within normal limits. S. Creatinine and Electrolytes were also normal. His IL-6 level could not be done due to limited facilities.

Table I - Markers from Day 19 - Day 21 suggestive of underlying secondary bacterial infection with sepsis, and cytokine storm.

\begin{tabular}{|c|c|c|c|c|c|}
\hline Day & $\begin{array}{l}\text { Total WBC } \\
\text { Count } \\
\left(4-11 \mathrm{~K} / \mathrm{mm}^{3}\right)\end{array}$ & $\begin{array}{ll}\text { CRP } & \quad(<10 \\
\mathrm{mg} / \mathrm{L}) & \end{array}$ & $\begin{array}{l}\text { Lactate } \\
(6-19 \mathrm{mg} / \mathrm{dL})\end{array}$ & $\begin{array}{l}\mathrm{LDH} \\
(135-225 \mathrm{U} / \mathrm{L})\end{array}$ & $\begin{array}{l}\text { S. ferritin } \\
(20-300 \\
\mathrm{ng} / \mathrm{mL})\end{array}$ \\
\hline Day 19 & 20.9 & 120 & 33.4 & 641 & 5820 \\
\hline Day 20 & 27.6 & 163 & 46.9 & 722 & 4941 \\
\hline Day 21 & 36.4 & 216 & 49.8 & 782 & 4830 \\
\hline
\end{tabular}


Meanwhile, his oxygen requirement increased to $15 \mathrm{~L}$ with non-rebreather mask on Day 20 and the patient was showing signs of air hunger with the respiratory rate increased to $33 / \mathrm{min}$. At this stage, he was labelled clinically as Severe Covid-19 Pneumonia. Seeing the upward trend of leukocytosis, CRP, lactate and LDH, Dexamethasone dose was reduced to $5 \mathrm{mg} 12$ hourly on Day 20 to prevent infective flare.HRCT chest was done on Day 20 which showed $80 \%$ lung involvement (Fig:2).His fever peaked at $103^{\circ} \mathrm{F}$ on Day 21 with marked tachycardia.Considering the extensive HRCT involvement and other investigation findings indicating a possible secondary bacterial infection, and his clinical condition deteriorating, decision was made to change the antibiotic and start Piperacillin/Tazobactam and I/V Linezolid (on Day 22). It was chosen on clinical judgment as the antibiotic sensitivity was not available due to negative blood culture. However, despite very high level of CRP and Ferritin, Tocilizumab was not given to the patient because of the high suspicion of secondary bacterial infection and sepsis, as shown by the very high lactate, $\mathrm{LDH}$ and total WBC count.2 days later,patient showed signs of improvement clinically with his respiratory rate coming down to 20/min.On Day 25, his temperature reached baseline for the first time since admission. He felt much better than before and said that he could "breathe easily". His oxygen requirement came down to $10 \mathrm{~L}$. His steroid was discontinued at this stage on Day 25, getting it for a total of 10 days. Repeat RT-PCR was given that day (Day 25) which came positive once again.

Nevertheless, his condition kept improving with his oxygen requirement reducing further. Repeat investigations showed markers coming towards normal value on Day 27 . He was finally removed from artificial oxygen on Day 30. CXR was done the next day (Day 31) which showed marked improvement (Fig: 3). With the markers on the decline and improved CXR,along with remission of all symptoms he was finally discharged with advice on the following day (Day 32). Patient was advised to stay in isolation for atleast 3 days after discharge and to be under follow up.
Patient came for follow-up 10 days later (Day 42 from symptom onset) with a repeat HRCT chest which showed huge improvement (Fig:4). CBC and other markers including CRP, ferritin, lactate and LDH were also reduced within normal limits.

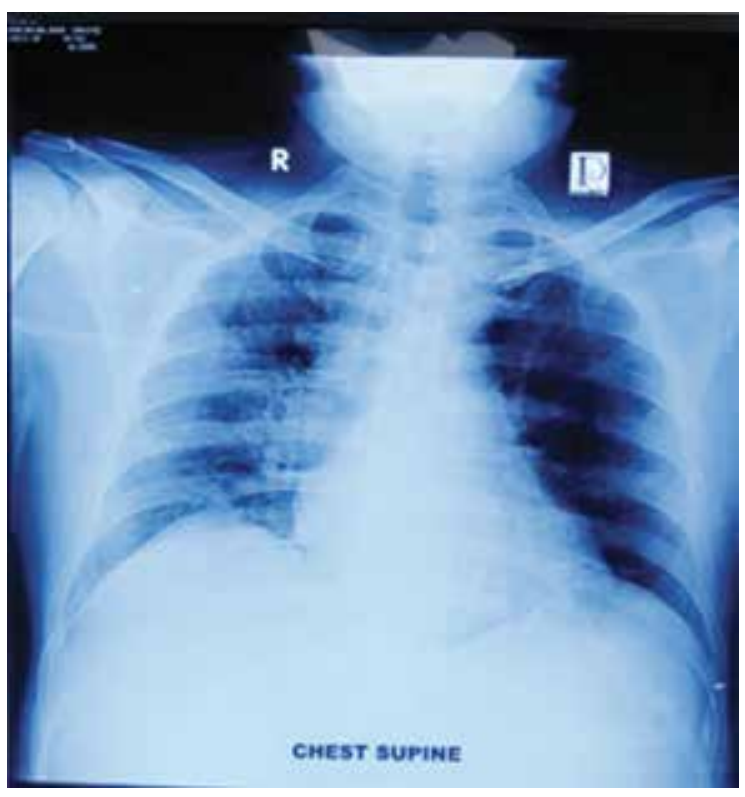

Fig:1 - CXR on Day 17 showing bilateral lung peripheral opacities

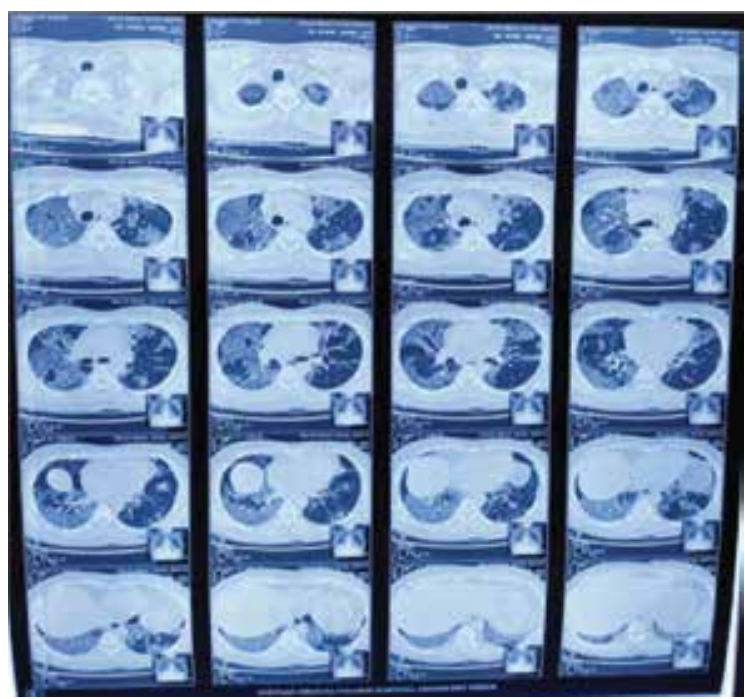

Fig:2 - HRCT chest on Day 20 showing bilateral extensive ground glass lesion, consolidations with intra and interlobular septal thickening as well as crazy paving lesion- Suggestive of severe Covid-19 pneumonia with around $80 \%$ involvement 


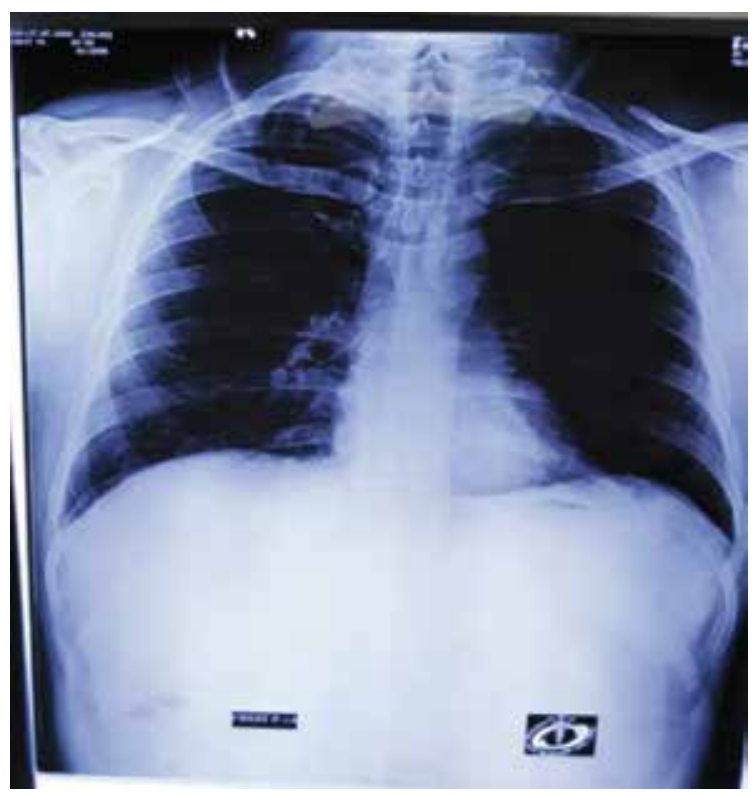

Fig:3 - CXR on Day 31 showing marked improvement

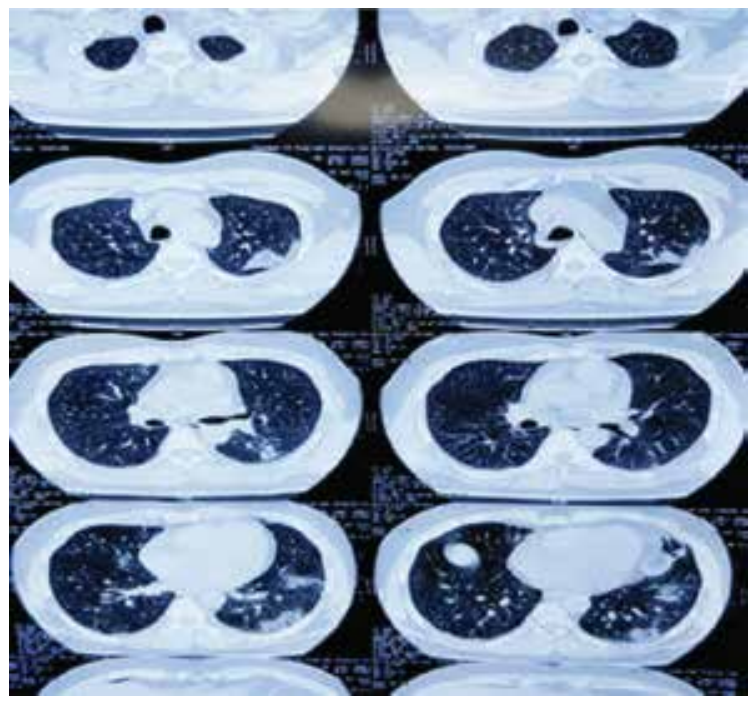

Fig: 4 - Latest HRCT chest showing gross improvement from the previous

\section{Discussion:}

People with the same infection may have different symptoms, and their symptoms may change over time. For example, one person may have a high fever, cough, and fatigue, and another person may have a low fever at the start of the disease and develop difficulty in breathing weeks later. All the symptoms of Covid-19 are non-specific, which means that they are also seen in some other diseases ${ }^{5}$. In our case, the patient developed cough at the onset, followed by low grade fever with difficulty breathing manifesting as a late presentation, which could be attributed to his early use of drugs at home.

An observational study at the Tongji Hospital in Wuhan came up with some patterns seen in the disease course of Covid-19. When SARS-CoV-2 infects a person, the lesions are not limited to the lungs. The virus enters through the airways by inhalation into the lung where it binds with Type-2 pneumocytes which contains ACE-2 receptors. From the lungs, it enters the blood and gets distributed to other organs of the body.This process includes the incubation phase and the early phase of the disease $^{6}$.In 7-14 days, viral replication usually stops, and our immune system takes over.

Our patient presented late to us, on Day 16 of his illness, without any previous HRCT. Pre-admission CXR revealed normal findings, but we believe an HRCT at that stage would be more rewarding. We delayed the HRCT by 4 days till symptoms progressed further to get the maximum information from the HRCT. We got $75 \%$ lung involvement in HRCT chest on Day 20.Patients at this phase usually begins to develop the hypercoagulable state. D-dimer is associated with severity of Covid-19. D-dimer in our case, however, always remained within the normal limit.It is believed that Covid-19 can activate coagulation cascade through various mechanisms, leading to severe hypercoagulability. Early anticoagulation may block clotting formation and reduce formation of microthrombus, thereby reducing the risk of major organ damages ${ }^{7,8}$. Thereby, we started the patient on anticoagulant from the beginning despite his normal D-dimer.Normal D-dimer values in a patient having such massive lung involvement is an unusual finding.

A meta-analysis of 3062 patients from 31 provincial level regions in China published in the Journal of Medicine Virology showed some patients presented with liver and renal functions abnormalities, which manifested as an increase in ALT,AST, and creatinine. So intense monitoring and evaluation of the function of important organs in Covid-19 patients should be considered $^{9}$. In our patient, ALT was raised but S.creatinine was normal.Biomarkers such as serum ferritin, CRP, LDH, procalcitonin have also got important prognostic values in Covid-19 evaluation. Higher CRP and LDH have been linked to unfavorable aspects of Covid-19 disease, such as ARDS development ${ }^{10}$, myocardial injury and death ${ }^{11}$. Regarding ferritin, Wu et al. showed that higher serum ferritin was associated with ARDS development. 
The increased levels of all these markers also seem to indicate the onset of a cytokine storm in patients ${ }^{12}$. Another emerging biomarker for Covid-19 course is interleukin6 (IL-6). In a study by Chen et al. $52 \%$ of patients had elevated IL-6 levels at admission. Increased IL-6 levels have been associated with increased risk of death ${ }^{12}$. In our case we could not do the IL-6 level.This was our limitation.

A meta-analysis of 3338 patients across the globe including USA, Spain and China showed bacterial co-infection in $3.5 \%$ of patients and secondary infection in $14.3 \%$ of patients with Covid- $19^{13}$. This is evidenced by an increased WBC count, high LDH, high procalcitonin,increased lactate and a positive blood culture. While neutrophilia could be attributed to the concomitant use of steroid in Covid-19, in our case, the count was sky high, which is unlikely to be due to steroid use. Also, our patient's LDH and lactate levels were also very high which all raise strong suspicion for an underlying secondary bacterial infection and sepsis. Confirmation by bacterial growth in culture could not be done however, as we did not get any organism in blood culture, possibly due to his very early use of antibiotics. So antibiotic sensitivity could not be seen, hence we had to start the most potent antibiotic on clinical judgement.

To test whether Remdesivir could help treat patients with COVID-19, a team of researchers of NIH carried out a randomized, controlled clinical trial enrolling 1,063 hospitalized adults with moderate to severe COVID-19 disease from across 10 countries. The analysis found that Remdesivir shortened the time to recovery. The median time to recovery was 11 days for patients treated with the drug compared with 15 days for those who received the placebo.The results also suggest that the drug may have some benefit in late use for surviving Covid-19. After 14 days, $7.1 \%$ of those in the group receiving Remdesivir died versus $11.9 \%$ of those in placebo group $^{14}$. Our patient presented on Day 16 at the hospital. Despite the late presentation, we chose to start Remdisivir on the benefit of doubt that it could be of benefit.

Approaches such as corticosteroids is considered to combat hyperinflammation. Broad immunosuppression in patients with overwhelming viral illness might be inadvisable. Beneficial anti-inflammatory effects should be weighed up against the potentially detrimental effects of inhibiting anti-viral immunity. Our patient received corticosteroid for a total of 10 days from admission.Although, earlier use of Tocilizumab in Covid-19 infection has been shown to be beneficial for survival in some studies, its use remains controversial. Administration of tocilizumab results in liver injury with a rise in ALT. Importantly, tocilizumab may be associated with opportunistic infections ${ }^{15}$. Regarding this in our patient, although decision about giving tocilizumab were being considered, it was eventually aborted considering the potential risk over benefit of opportunistic infection as the patient's total count, LDH and lactate levels were significantly raised.

Our case was RT-PCR positive on Day 25 of his disease. However, a positive RT-PCR result does not prove that replicating virus is present. It simply proves that its genetic material, the RNA strand, or the dead viral remnant is present, which can also be present without any active viremia.Usually RT-PCR becomes negative in most patients after 2-3 weeks, but a small percentage continues to be positive for much longer. There are some concerns that if you can still find RNA, some live virus could be present. But there are reverse arguments that the amount of virus is negligible to be transmissible ${ }^{16}$.

WHO's updated criteria for end of isolation states that for symptomatic patients, isolation should be kept for at least 13 days from symptom onset if symptom duration is less than 10 days. If symptom duration is more than 10 days, isolation should be maintained during the symptoms and an extra of at least 3 days after symptoms subside ${ }^{17}$. So, we advised the patient to stay in isolation for at least 3 days at home after discharge.

\section{Conclusion:}

We selected this case for publication to highlight some important issues. We wanted to show that although Covid-19 is a viral illness, it can present with scenarios suggesting a possible secondary bacterial infection and even sepsis. The beauty of this case lies in the logical courage to manage the patient in general ward without shifting to higher facilities like HDU, despite possible sepsis. We want to highlight that such patients can be managed in general ward setting with adequate monitoring if the patient does not progress to shock requiring ionotropic support.Another interesting thing about this case was that despite the cytokine storm, we decided against use of any immune modulatory agents due to the risk of infective flare, which eventually yielded good results. We also decided against convalescent plasma therapy because it was already past 2 weeks by the time the patient came to 
us. With all these crucial decisions taken, the patient eventually managed successful recovery in ward settings.

Another unusual feature of this patient was the normal D-dimer level with disproportionately massive lung shadows which shows the vast array of possibilities of presentation of Covid-19.The positivity of RT-PCR on Day 25 also teaches us that though the virus may stop replicating in the host, its remnants may stay inside for an extended period.We are still at very early stages of understanding this new disease. Although, this disease is presenting in various ways in populations across the world, we have managed to track some set pattern of disease progression. This, along with expert opinions from experienced consultants, can help us combat this disease going forward.

\section{References:}

1. Zhu N, Zhang D, Wang W, et al. A Novel Coronavirus from Patients with Pneumonia in China, 2019. N Engl J Med. 2020; 382(8): 727- 733. https://doi.org/10.1056/ NEJMoa2001017, PMid:31978945 PMCid:PMC7092803

2. Abdelli, I., Hassani, F., Brikci, S. B., \& Ghalem, S. (2020). In silico study the inhibition of Angiotensin converting enzyme 2 receptor of COVID-19 by Ammoidesverticillata components harvested from western Algeria. Journal of Biomolecular Structure and Dynamics, 1-17. https://doi.org/10.1080/07391102.2020.1763199. PMCid:PMC7232889

3. Mehta P, McAuley DF, Brown M, et al. COVID-19: consider cytokine storm syndromes and immunosuppression. Lancet. 2020; 395(10229): 1033- 1034. https://doi.org/ $10.1016 / \mathrm{S} 0140-6736(20) 30628-0$

4. Shimizu M. Clinical features of cytokine storm syndrome. In: Cron R, Behrens E editors. Cytokine Storm Syndrome. Cham: Springer (2019). 31-42. doi: 10.1007/ 978-3-030-22094-5_3, https://doi.org/ 10.1007/ 978-3030-22094-5_3, PMCid:PMC6371477

5. Zhang W, Du RH, Li B, et al. Molecular and serological investigation of 2019-nCoV infected patients: implication of multiple shedding routes. EmergMicrob Infect. 2020;9:386-389. doi: 10.1080/22221751. 2020.1729071. https://doi.org/10.1080/22221751.2020.1729071, PMid:32065057 PMCid:PMC7048229

6. Working Group of 2019 Novel Coronavirus, Peking Union Medical College Hospital. Diagnosis and clinical management of Severe Acute Respiratory Syndrome Coronavirus 2 (SARS-CoV-2) infection: an operational recommendation of Peking Union Medical College Hospital (V2.0). EmergMicrob Infect. 2020;9:582-585. https://doi.org/10.1080/22221751.2020.1735265, PMid:32172669 PMCid:PMC7103730

7. Lin L, Lu LF, Cao W, et al. Hypothesis for potential pathogenesis of SARS-CoV-2 infection-a review of immune changes in patients with viral pneumonia. EmergMicrob Infect. 2020. https://doi.org/10.1080/ 22221751.2020.1746199, PMid:32196410 PMCid:PMC7170333

8. https://onlinelibrary.wiley.com/doi/full/10.1002/jmv. 25884.

9. Wu C, Chen X, Cai Y, et al. Risk factors associated with acute respiratory distress syndrome and death in patients with coronavirus disease 2019 pneumonia in Wuhan, China. JAMA Intern Med. 2020. https://doi.org/10.1001/ jamainternmed.2020.0994. PMid:32167524 PMCid:PMC7070509

10. Deng Y, Liu W, Liu K, et al. Clinical characteristics of fatal and recovered cases of coronavirus disease 2019 (COVID-19) in Wuhan, China: a retrospective study. Chin Med J (Engl). 2020;133(11):1261-1267. https://doi.org/ 10.1097/CM9.0000000000000824, PMid:32209890 PMCid:PMC7289311

11. Chen N, Zhou M, Dong X, et al. Epidemiological and clinical characteristics of 99 cases of 2019 novel coronavirus pneumonia in Wuhan, China: a descriptive study. Lancet. 2020;395(10223):507-513. https://doi.org/ $10.1016 / \mathrm{S} 0140-6736(20) 30211-7$

12. Lansbury L, Lim B, Baskaran V, Lim WS. Co-infections in people with COVID-19: a systematic review and meta-analysis. J Infect 2020 (ig9, 7908424). https://doi.org/ $10.2139 /$ ssrn. 3594598

13. https://www.sciencedirect.com/science/article/pii/S12 01971220305804

14. Beigel JH, Tomashek KM, Dodd LE et al; ACTT-1 Study Group Members. N Engl J Med. 2020 May 22. doi: 10.1056/NEJMoa2007764. Online ahead of print. PMID: 32445440. https://doi.org/10.1056/NEJMoa2007764, PMid:32445440 PMCid:PMC7262788

15. US Food and Drug Administration. ACTEMRA (Tocilizumab) Injection, for Intravenous or Subcutaneous Use. US Food and Drug Administration; 2017.

16. Doctors Wonder What to Do When Recovered COVID-19 Patients Still Test Positive - Medscape - Jun 09, 2020.

17. World Health Organization. Clinical management of COVID-19 (Interim Guidance) https:/www.who.int/ publications-detail/clinical-management-of-covid-19, published 27 May 2020. 